\title{
A PRACTICAL TRANSIT NETWORK DESIGN METHOD
}

\author{
W R Duff-Riddell \\ Institute for Transport Technology, Department of Civil Engineering, University of Stellenbosch, \\ Private Bag X1, Matieland, 7602, South Africa.
}

\section{INTRODUCTION}

Although many aspects of transit (public transport) system design and operation have been extensively researched, there appears to have been very little published on the practical design of transit lines, especially in systems involving more than one mode and diverse vehicle fleets. (One of the more comprehensive treatises on this subject is the work by Hasselström, 1981.) Computerised models which have been developed for this purpose are often highly specialised and very expensive to purchase or incur substantial set up costs or both. This paper describes, in general terms, such a practical design process using general transport network models and readily available office software. It is shown how an adaptation of the Emme/2 transport network model, (or other similar packages such as SATURN,) can be used in conjunction with a transit resource database and a goal-programming model to produce a set of fully described transit lines on a general transport network. The process, currently only experimental, is shown in flowchart form in Figure 1.

The basic inputs to the Emme/2 model are the general transport network, and the auto and transit demand matrices. Using the "auto assignment" option of Emme/2, and taking the auto traffic into account, modified volume delay functions (VDFs) are used to aggregate or "focus" transit demand, onto routes with sufficient demand volume to be operated viably in terms of system constraints. The assignment process, initially a "free," or "user optimal" assignment of transit trips, is increasingly constrained in subsequent iterations, until practical viability of the transit system is achieved.

After focusing, the assignment results are converted, by means of a Microsoft Visual Basic ${ }^{\mathrm{TM}}$ program written for the purpose, into a set of potential transit routes. Goal programming (GP) is then used to assign the available fleet to these potential routes by seeking an optimal vehicle allocation for the demand on the proposed routes. The exact form of the GP model is dependent on local planning objectives, but includes information on the potential routes, derived from the network model, and information on the fleet, such as the number and operating characteristics of all available transit vehicles. Optimisation objectives, will typically include; satisfaction of demand, vehicle utilisation factors, total costs, minimum service frequencies, (and thus waiting times,) maximum fares and subsidy rates. Other objective variables can be introduced as required. Nonviable route data is used for further focusing if necessary. The process is repeated until all constraints are satisfied, or no further objective function improvement can be achieved. 


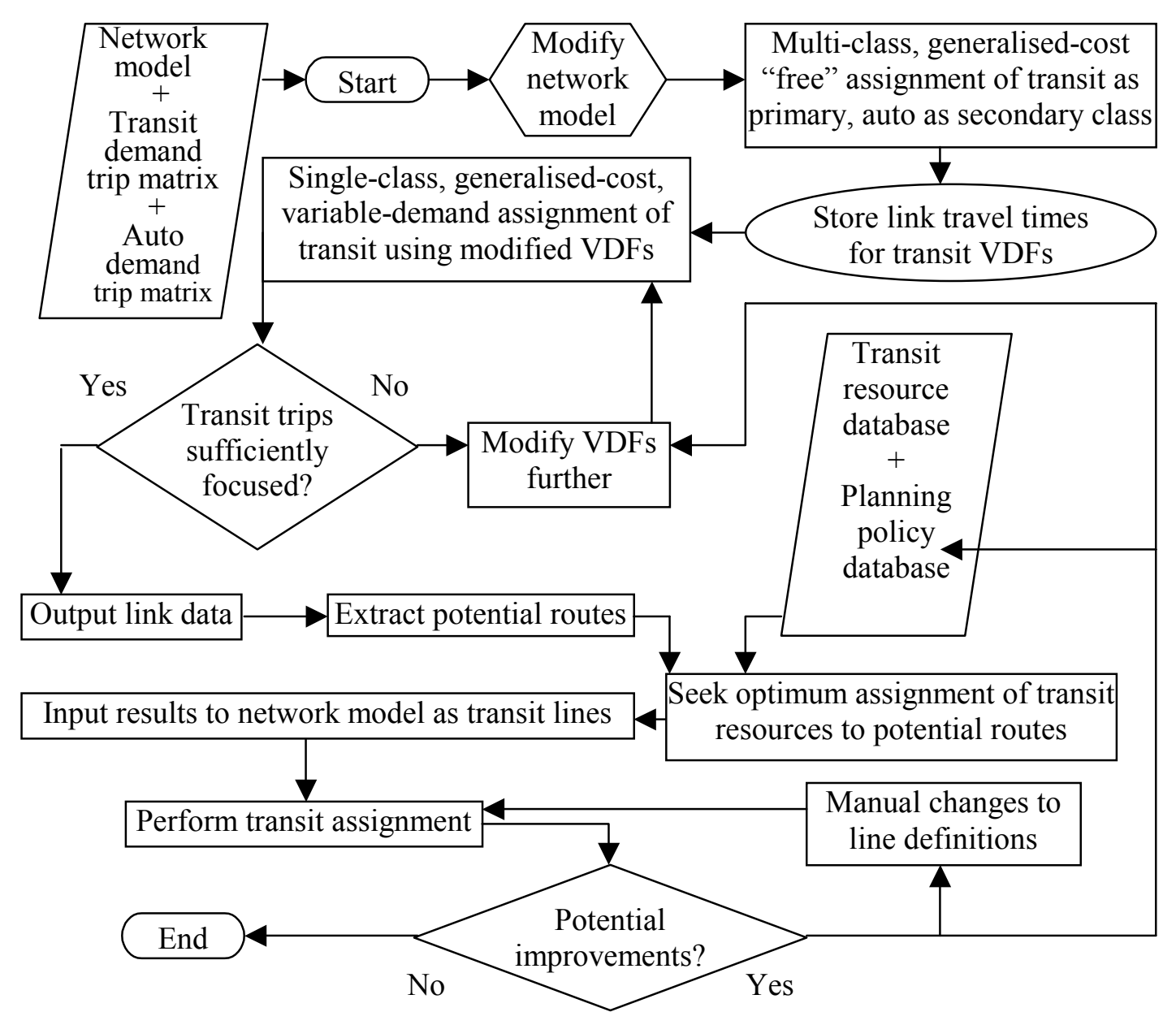

Figure 1: Flowchart of transit network design method.

\subsection{SOME TERMINOLOGY USED}

The following terms may not be familiar to the reader:

Zone _ - A subdivision of the area to be modelled, usually of more or less homogeneous land use type, such as residential, industrial or commercial.

Centroid - The hypothetical centre of a zone where all trips from and to the zone originate and terminate. A centroid is a special form of node.

Node - A regular node is a point representing a connection between two or more links. A centroid node is as described above.

Link - Typically, a road or rail segment. Each direction, but not necessarily lane, constitutes a separate link. Bus-only lanes are modelled as separate links.

Assignment - A process in which trips are assigned to the transport network, usually in such a way that no one can improve their travel time by choosing a different route. Multi-class assignment superimposes different user groups on the network.

Class - A group (or class) of users having similar perceptions of the cost of using the network.

Volume delay function - (VDF) A mathematical representation of the travel time on a link as a function of the number of vehicles using the link and the vehicle capacity of the link.

Focus - A modelling process in which trip makers are encouraged to select common routes. In other words, trip makers gather together on some route, not first choice to most of them, in order to justify a transit service. 


\section{THE NETWORK MODEL}

This discussion assumes that an Emme/2, or similar, network model is available. If an integrated, multi-modal transit system is to be achieved, the network model for the planning of a transit system must reflect all possible transit facilities, railway lines, ferry services and HOV lanes. All significant interchange facilities, railway stations for example, must be included, by representing them as nodes connected to at least two modes so that mode changes can be modelled. The modelling process may show these facilities to be redundant or significantly under-utilised in an optimal transit system. Similarly however, currently unused infrastructure may prove to be key components of the optimal system. At this stage, it is assumed that all roads and dedicated transit facilities in the modelled network are available to transit. Where minibus-taxis or perhaps even conventional taxis will form part of the fleet, it may be necessary to increase the level of detail present in the network model.

There are four aspects of the network model requiring particular attention:

\subsection{VEHICLE OCCUPANCY}

The travel time on a network link is a function of the link capacity and the number of vehicles using the link. The number of vehicles is dependent on the number of people travelling and the average vehicle occupancy rate. For car travel, the average occupancy rate is often determined on an origindestination basis. In a multi-modal transit system, there is no standard vehicle occupancy for transit vehicles and occupancy is not origin-destination dependent, except possibly on express routes. It is thus necessary to use a global vehicle occupancy rate for transit vehicles.

In South Africa, the high prevalence of mini-bus taxis makes this vehicle a useful basis for a global vehicle occupancy rate. Assuming the mini-bus taxi to have a passenger car equivalent of about 1 , then a vehicle occupancy rate of 14 passengers per vehicle seems reasonable. Using a 14-passenger occupancy rate, a bus carrying 60 passengers will be perceived as being the equivalent of five standard motor cars. On the basis that increased passenger numbers increase the number of stops, boardings and alightings, the interference between transit vehicles and other traffic can be expected to increase as the number of passengers increases. Concurrently, the operating characteristics of larger vehicles also result in a higher passenger car equivalent value. This estimate of vehicle occupancy is far from perfect, but provides some approximation of the impact of high transit demand on network traffic patterns. The volume delay functions of the transit-only road links and non-road modes need to be constructed keeping this average occupancy in mind.

\subsection{VOLUME DELAY FUNCTIONS}

The link travel time for non-transit traffic is determined using a "volume delay function." This is a mathematical model of the travel time on a link, which incorporates, the minimum possible travel time, the vehicle demand, the vehicle occupancies and calibration parameters for the specific link type. Transit-only links are usually modelled on scheduled travel times. In this process however, volume delay functions must be developed for all dedicated transit links. These links must be given volume delay functions that reflect the best reasonable travel time of the links, normally approximately constant up to the modal capacity limit. (Where congestion is certain not to occur, a constant travel time will suffice instead of a proper volume delay function.) Where congestion may occur and a BPR (Bureau of Public Roads) type volume delay function, as shown below, is applied, the use of a high value of $\alpha$, around 10, produces a suitable curve. 
BPR Volume Delay function:

$$
t(v)=t_{0} \cdot\left(1+\left(\frac{v}{c}\right)^{\alpha}\right)
$$

Where:

$$
\begin{array}{ll}
\mathrm{t}_{0}=\text { Minimum travel time on link. } & \mathrm{v}=\text { Vehicle volume on link. } \\
\mathrm{c}=\text { Vehicle capacity of link. } & \alpha=\text { Calibration parameter. }
\end{array}
$$

A universal vehicle occupancy rate is used in the multi-class assignment and the volume delay function must be written in terms of this occupancy rate. The vehicle volume is determined, within Emme/2, as the person-trips divided by the average vehicle occupancy rate. Thus, the capacity of non-road modes such as rail must be given as the actual link capacity divided by the same occupancy rate. For example, if an occupancy rate of 14 people per vehicle is used, and a railway line sees a demand of 6000 people, then Emme/2 will set $\mathrm{v}$ as $6000 / 14=429$. Thus, if the line has a capacity of 10000 people, then c must be entered as $10000 / 14=714$ to correctly model the effect demand on travel time.

This volume delay function is a very rough approximation of reality because passengers are only required to wait at their point of boarding, not at subsequent links and thus, waiting time cannot be included. If waiting times are considered relevant for some modes, in relation to the other modes, dummy links connecting the rest of the network to the mode access nodes can be used to simulate the waiting time. This technique could be applied to all transit mode access points but this would require very extensive model changes. The main objective is to achieve a relative travel time for each available mode since all transit modes have some waiting time penalty as against the auto mode.

\subsection{GENERALISED-COST}

Generalised-cost is a measure of the total cost of making a trip, including actual travel time and such measures as, waiting time, fare, toll fees etc. In the Emme/2 modelling process, the generalised-cost is a "constant function" of the link length and is assigned to one of the link user variables. In this route-planning model it applies only to the transit users. In a road-only environment, the generalised-cost can be the free-flow travel time, a function of link length and speed limit. The use of link length results in transit trips favouring the physically shorter routes whilst the auto traffic diverts to longer routes in order to maintain the travel time equilibrium. If a certain link or corridor is to be encouraged or discouraged then it may be given a generalised-cost which promotes this policy. For example, multiplying the link length by some fractional constant will promote its use whereas adding some constant to the link length can make it unduly "expensive" to use.

If non-road modes are present, then a mode fare factor per unit length may be necessary in the evaluation of the generalised-cost. In this way, user choice of a cheaper mode over a quicker one, depending on their value of time, can be modelled. To do this, the monetary value of a time-unit is determined and then converted to the equivalent time value of a monetary unit. The concept is demonstrated in an example: 
Assume two routes between $\mathrm{A}$ and $\mathrm{B}$, one bus and one rail with the following attributes:

\begin{tabular}{|l|ll|}
\hline & Bus & Train \\
\hline \hline Fare per $\mathrm{km}$ & $\mathrm{R} 0.50 / \mathrm{km}$ & $\mathrm{R} 0.30 / \mathrm{km}$ \\
Km & $20 \mathrm{~km}$ & $31 \mathrm{~km}$ \\
Average speed & $30 \mathrm{~km} / \mathrm{hr}$ & $45 \mathrm{~km} / \mathrm{hr}$ \\
\hline
\end{tabular}

From these attributes, it can be seen that the following values apply for the alternative routes between $\mathrm{A}$ and $\mathrm{B}$ :

\begin{tabular}{|l|l|c|}
\hline & Fare & $\begin{array}{c}\text { Travel time, determined by } \\
\text { volume delay function. }\end{array}$ \\
\hline Bus & R 10 & 40 min \\
\hline Train & R 9.30 & $41 \mathrm{~min}$ \\
\hline
\end{tabular}

Let the value of in-vehicle time be R12.00 per hour for a typical transit-using commuter. Then the time value of a Rand is 5 minutes. Thus, the total time value of travelling by bus is $40+\mathrm{R} 10 * 5=$ 90 minutes, and for train is $41+\mathrm{R} 9.30 * 5=87.5$ minutes. Assuming that the value of time will be included in the link attribute then, in the example, the fixed-cost (FC)for a link will be:

FClink $=$ Length $*$ fare per $\mathrm{km} *$ time value of a Rand

This gives:

For a bus link: $\quad$ FCbus $=$ length $* 0.5 * 5=$ length $* 2.5$

And for rail: $\quad$ FCrail $=$ length $* 0.3 * 5=$ length $* 1.5$

The generalised-cost (GC) will be:

GClink $=$ Link travel time + Weight $*$ FClink

If, as above, the value of time is included in the link attribute, then the fixed-cost weight-factor will be 1 although it may be altered to emphasise either travel time or distance. Time based costs being dominant to the operator and thus in the fare, a fractional weight seems to be sensible, thus avoiding a distance dominated route determination.

\subsection{MODES}

The network modelling process incorporates a number of distinct modes of travel; walk, car, transit, auxiliary auto, auxiliary transit etc. These are used to allow certain categories of user access to certain parts of the network by indicating which defined modes are allowed on each link. These mode and link definitions need to be changed. All links, irrespective of mode, are defined as supporting the auto mode. This is necessary to conform to the restrictions of the Emme/2 modelling package in which only the auto mode has unrestricted access to all links. From the Emme/2 User's Manual page 4-354:

The subnetwork accessible to each class is defined either by the auto mode or by an auxiliary auto mode. Note that a subnetwork defined by an auxiliary auto mode contains only the links where both that mode and the auto mode are allowed. The demand for each class is assigned to paths that are restricted to the relevant subnetwork.

All conventional auto links are defined as being auxiliary auto links thus restricting the non-transit road traffic to the original real road network. This allows the assignment of the multi-modal transit and regular auto traffic to the network concurrently. Transit users have access to the entire network and can "change" mode when this improves their generalised travel cost. The modifications can be quite easily implemented in Emme/2 using a macro. 


\section{THE DEMAND DATA}

Travel demand data is required for any form of transport modelling. Some demand data will be available with the original network model although more comprehensive data may be required. At the bottom of the range of choices for data on transit demand, is the existing pattern of transit usage for each main period, (AM and PM peaks, off-peak etc.) This data has the drawback of not making any allowance for the potential users for whom the existing service provides no acceptable alternative. The next level of data desirability is a fixed demand matrix, based on a region wide survey, taking into account only time-based origin-destination demand.

The demand data complexity can be built up until a full matrix of demand functions is obtained, based on stated and revealed preference studies, which include aspects of safety, comfort, service frequency, fare and a whole range of other issues for each possible planning period. This is expensive to obtain but necessary when attempting to attract choice users on the one hand and while catering for impoverished captive users who value money more highly than time and comfort on the other. Such a demand function matrix will allow a rough modal choice component in the transit modelling process. For the purpose of this paper, a basic, temporal, origin-destination demand matrix is assumed available.

\section{THE FOCUSING PROCESS}

The network model, that describes the transit and auto networks, and an origin-destination demand matrix are now available. It is now necessary to assign the travel demand to the network. The first step is a generalised-cost, multi-class assignment.

\subsection{MULTI-CLASS ASSIGNMENT}

In this assignment, all network users are assigned to the network in an equilibrium assignment of vehicle trips using the relevant occupancy rates. The interaction of transit and non-transit traffic is modelled and the network wide influence of this interaction can be seen. The transit demand is assigned as the primary class - this means that the transit users can choose any route they wish and the auto demand as the secondary class. If the original model includes additional traffic, which affects network performance, this can be included as applicable. (Research is still required to investigate the possibility of assigning captive riders as "Additional demand" and choice riders as the primary-demand so that the decision to establish a route can be made based on need.)

Travel-time and travel-distance minimisation are inherent in the generalised-cost assignment process. Transit users will select the best path in distance, because the generalised-cost includes the link lengths, as well as in time and, if applicable, fare-cost terms. Auto users will select the shortest time path available to them given that transit will get preference on the shorter paths. This supports three optimisation considerations:

1) The operators will minimise their operating mileage and route travel time and thus costs. (The vehicle mileage between depot and route end, is another issue.)

2) The transit customers will travel by the shortest and cheapest path.

3) Transit is favoured over private travel by being given preference on the "best" routes in the planning process.

From this assignment, the travel time of every link is saved to a link user-attribute, say LUA1. A copy of the model is made, this is known as a new scenario in Emme/2 parlance. Two copies are made if sufficient demand exists between origin-destination pairs to justify dedicated services. One copy reflects express and the other general transit services. The process is similar for the two groups 
but by separating express route passengers, transfers to the "express line" are prevented. Some changes are made to the copy(s) as discussed in the next section. The rest of the discussion focuses on the regular rather than express routes.

It could be argued that the multi-class assignment is unnecessary and that a free assignment of the transit trips using the link travel times from a normal auto assignment would be adequate. This approach was adopted by the Ministry of Transport in Israel to investigate route alternatives (Elgar \& Kfir 1992.) It should be remembered however, that once even a few hundred commuters an hour are using a road based transit link, there can be significant impacts on the overall traffic distribution which affect both private and transit traffic. This is of particular relevance when corridor development is being considered, especially when alternative mode choices exist for transit users.

\subsection{SINGLE-CLASS ASSIGNMENT AND FOCUSING}

\subsubsection{The first single-class assignment}

The new scenario is now modified so that all the volume delay functions reflect the link travel time derived in the multi-class assignment. This is done by setting the link volume delay functions (fd\#\#,) to the link travel time stored in link-user-attribute 1 (LUA1); For example: fd50=LUA1. A single-class, generalised-cost, (variable-demand if data is available,) assignment is now performed with this modified scenario. The generalised-cost remains as before. Only the transit demand is assigned and this without a vehicle occupancy matrix; i.e. each trip maker is assigned as an individual. The result of this assignment is similar to the previous one, inasmuch as that the same fundamental link volumes pertain albeit that the vehicles are now split into trips and the private traffic is only represented by virtue of travel time. This assignment still assumes that there is a "perfect" transit system in which there is negligible waiting time or delay. This is the best trip time that any traveller could hope to achieve on the network using any mode, including private auto. In the case of a variable demand assignment, the actual number of trip makers and their modal choice will be influenced by travel time and fare cost factors where included in the generalised-cost.

\subsubsection{Focusing}

At this stage, the model will probably have many links with insufficient demand in either direction to justify any sort of transit service. The low demand problem is minimised by focusing the trips onto busier routes wherever possible. This is achieved by replacing the volume delay function of the low volume links (and their reverse link) with one carrying a heavier penalty; for example: fd51=10*LAU1. Focusing the trips is an iterative process. The travel cost on the network links with low volume is raised in consecutive assignments, each basing the revised cost on the previous assignment results. The much higher minimum-demand of non-road-mode routes, rail for example, may be taken into account by including mode criteria in assigning the volume delay functions. It will fall upon the planner to decide on the specific focusing strategy. The travel times derived from the multi-class assignment are not significantly affected because typically, the focusing process eliminates trips from routes where the influence of transit is minimal in the first place. Similarly, the routes gaining trips are normally only going to see an increase of one or two vehicles, if any, as spare capacity is taken up.

The focusing process operates as follows: After the first single class assignment, links with zero or one trip are assigned an increased cost, say $\mathrm{C} 1$, and a new assignment is performed. Then using the results of the second iteration, links with zero or one trip are assigned a higher penalty, say C2; links with two trips are assigned a cost of $\mathrm{C} 1$ and the assignment repeated. Subsequent iterations raise the cost of a particular number of link trips, with the least number of trips presenting the highest trip cost. The process is repeated until successive assignments show no change in link volumes or the minimum link volume is considered adequate to justify a transit route. 
The increasing cost approach is realistic in the transit environment where it occurs, either as a high fare, or as a long headway. Where there are no alternative routes, users must either pay the higher cost, forego the trip or change mode. Where alternative routes do exist, users will choose the more heavily utilised, and thus "cheaper," routes providing that the ultimate cost of doing so is to their benefit. Using this iterative approach will tend to provide a more realistic set of route choices than the alternative of using one step in which a heavy penalty is applied to all links carrying less than say twenty-five trips.

The concept can be extended to assign heavy penalties to links, which are not to carry transit by virtue of their link type. This is preferable to taking the links out of the model-network because there may be circumstances in which a real-network modification is justified - especially where transit promotion is a priority. This will show up when trips are not diverted, even when a high penalty is imposed on a link or set of links. One could also lower the penalty on links on which transit is particularly desired, for corridor development for example. This reduced penalty would have to be backed by subsidy, transit right-of-way or high service frequency to match the reduced penalty in reality. Sometimes, a route may be operated for "political" reasons. In this case, the travel-time volume delay function is retained on links on the prescribed route irrespective of low demand - trips may then be encouraged to transfer from surrounding links during the focusing process, thus improving utilisation of the compulsory route.

Identifying the links on which the volume delay functions are to be modified requires some consideration. The maximum one-way volume, the two-way volume or a function of the two-way volume and the ratio of the forward and reverse volumes could be selected. If sufficient data is available, the link volumes for several study periods can be combined. Utilising time-aggregated data has the advantage of producing routes "common" to all time periods. This makes it easier for travellers to use the transit network. Various combinations of these options result in different focusing strategies and must be evaluated by the planning team in terms of local conditions and objectives. Ultimately, network coverage is dependent on the potential demand for transit and the focusing process criteria utilised.

It is recognised that the degree of accuracy in the routing of the transit vehicles resulting from the network model is dependent on the number of zones employed and the completeness of the network model. It is unusual to model larger cities with the degree of accuracy required to fine-tune the routes of road based transit vehicles. Such local routing would thus have to be performed separately or the network model upgraded to include all possible routes and as many zones as necessary to realistically describe the access requirements of transit users. Where a maximum walking distance is specified, the zones should not exceed that distance in radius. In any event, the Emme/2 results provide a fair representation of how the transit routes should be structured without any further processing. The process can however be taken considerably further.

\section{THE ROUTE EXTRACTION PROCESS}

Once trip focusing is complete, the link and reverse link volumes are copied to link user-attributes and the link data is output to text file. The Emme/2 assignment results are not used directly, because link data is needed later to construct and input transit lines for testing and graphic display in Emme/2. The data is however not quite suitable as it is output by Emme/2 for the following reason.

Centroids usually represent hypothetical zone centres in which all zone trips originate or terminate. The impedance of the links connecting the centroids to the network reflects the average impedance experienced by trips starting or ending in the zone as they access the real network at the regular node end of the centroid link. Thus, the Emme/2 definition of centroids is such that trips may not 
pass through them. Emme/2 does however model trips as physically starting or ending at centroids, which may have several entering and leaving links. This gives rise to link volumes, which are perceived by the route extraction process as trips passing through the centroid. This problem is dealt with by eliminating all centroid links from the route extraction input data. If the centroids are numbered as a unique range, (e.g. 1 to 200,) which is the normal approach, this is a simple process and thus ensures that the transit routes follow the "real" network.

The basic route extraction process operates in the following way, each step recording the length and travel time of the links included in the path:

1) Find the network link with the highest outstanding demand. (See note below.)

2) Find the connecting link, of the same mode, with the highest demand.

3) Follow the trail until either the demand drops below the minimum requirement, or a mode change occurs.

4) Go back to the first link and track the links in the other direction.

5) Find the lowest viable demand on the links in the set. (Based on the available fleet and policy decisions.) This is the supply volume for the route.

6) Subtract this supply volume from the outstanding demand for each direction on all the links in the set. (This assumes a route to be bi-directional.) Excess capacity on a link is reflected by a negative demand in the database control field.

7) Save the link-set and start the process again with the revised network link volumes.

8) Repeat until all links have non-positive demand.

NOTE: The same alternatives can be used for route selection as discussed previously; i.e. multiple periods or two-way volumes, although actual peak-direction volumes must be used for route capacity specification. These refinements result in a higher overall utilisation pattern for the vehicles and need to be evaluated in terms of design policies. Although they have not yet been implemented, some restrictions on backtracking are possible, as are rough estimates of the boarding and alighting time on each link for each route to give a more accurate estimate of overall route travel time. This latter may be better implemented as a global factor however, as processing time will be considerably increased when volume changes at every node must be evaluated.

This process can be practically demonstrated to work effectively on very small networks but is less clearly effective in complex networks. A full origin-destination analysis of a grid network in which routes generated by this method were compared against a more conventional grid of transit lines was undertaken. Results showed concurrent improvements in vehicle kilometres and total transfers required when only one vehicle type was employed. Allowing the use of different vehicle types on different routes, the method further gave improvements in operating costs where smaller vehicles were presumed to be cheaper to operate. (That the approach is guaranteed to produce such improvements has not yet been established.)

An example of a small network and the core route extraction results are shown in Figure 2. Complete results from the extraction process, not shown here for lack of space and relevance to the example, include the node sequence and estimated travel time and travel route length. If there were trips between all possible origin and destination pairs, 15 return lines, (e.g.1-2-1, 6-5-7-8-7-5-6, etc.), would be required to eliminate all transfers. However, it can be seen that three lines, with very little spare capacity, will suffice to cover all trip combinations with a maximum of one transfer required of any user. Looking at the route between centroids 2 and 6 via intermediate nodes 4 and 5: Providing 90 seats will cover all the trips leaving centroid 2 and arriving at centroid 6 whilst ensuring that no line with less than 20 seats is required to satisfy trips in the primary direction, although some passengers will have to transfer. 


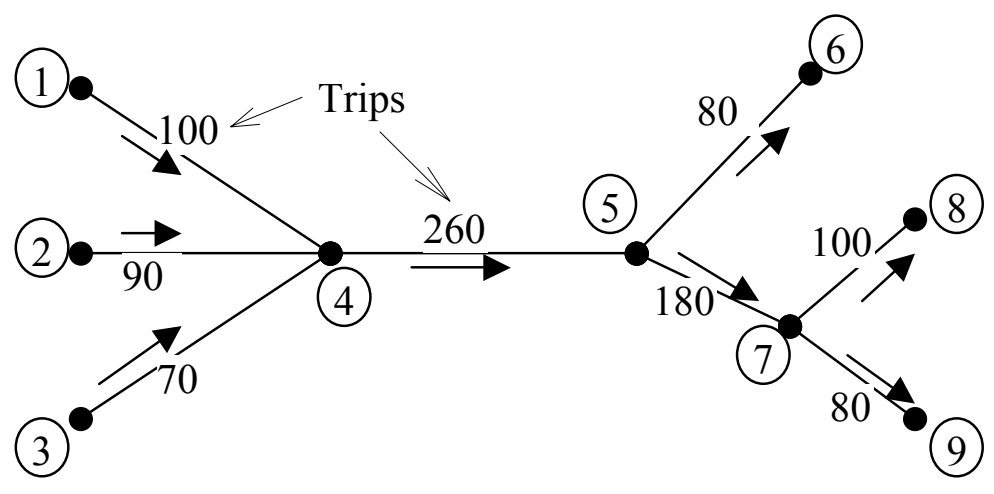

\begin{tabular}{|c|c|c|}
\hline From & To & Supply \\
\hline 1 & 8 & 100 \\
\hline 2 & 6 & 90 \\
\hline 3 & 9 & 80 \\
\hline
\end{tabular}

Centroids: $1,2,3,6,8,9$.

Regular nodes: 4,5,7.

Trips: link volume

travelling from left to right.

Figure 2: An example network with a table of potential routes resulting from extraction process.

(Based, in this case, on a minimum viable line capacity of 20 trips.)

In reality, it is rare for there to be sufficient demand between any two centroids to justify a direct service and thus many transit users are likely to have to make at least one transfer. (Research is still required into a "value of transfer" so that the cost of operating a line can be compared with the saving in transfers.) If there is proportionality between origin and destination volumes, (i.e. if $100 / 260$ of the trips from node 1 go to node 8 ,) then the process also minimises the number of transfers required with the given number of lines.

In South Africa, with many captive riders, providing accessibility probably outweighs the inconvenience of a transfer, which seems preferable to no transport at all. Maximised coverage through efficient use of resources thus seems to be a more logical primary focus of optimisation than transfer minimisation. By ensuring optimum vehicle utilisation, increased coverage is possible with the same fleet, thus improving the likelihood of actually being able to make a trip by transit, albeit at the possible cost of additional transfers. Research will be continued into developing a rigorous but practical method for ensuring the extraction of the optimum set of routes in terms of both goals.

The result of the process is a table of potential routes defining the length, approximate travel time, demand, and node sequence. Data on minimum terminal times and other pertinent factors can be appended manually, either by default on all routes or route by route. There is also a record of the number of times each link and node has formed part of a route and whether or not nodes have acted as route start and end nodes. This information is used to examine the siting of infrastructure. It is still however, not possible to say with any certainty that all these routes can be operated. To investigate this, the goal-programming model is used.

\section{THE GOAL PROGRAMMING MODEL}

Associated with the network model and the resulting output, is a database of the transit fleet and regional policy considerations. The fleet information will include approximate operating cost per kilometre and per hour, fuel type and consumption rate, capacity, minimum feasible headway, desired load factor, exhaust emission data, and so on, for each vehicle type, along with the number of such vehicles available. The policy data will include preferences for fuel type, average route frequencies, pollution targets and so on. These policies need to be weighted or prioritised in terms of local objectives.

The potential-route data and fleet database are now brought together in a goal programming model which seeks to match the fleet to the routes within the constraints and policy goals specified. A weighted goal-programming model, which works by minimising the undesirable deviations from 
the various objectives, has been used. Other goal programming modelling approaches, such as MinMax or Lexicographic (Romero, 1991) can also be used with some adaptation of the model shown below. The choice of approach will depend on the goals and objectives and their relative importance in the specific project. Consequently, this is the phase of the project most subject to interpretation and manipulation.

The modelling language, LINGO (Lindo Systems) has been used. This language has a model generator and thus requires only a template model, irrespective of the number of routes and vehicles to be evaluated. All data and parameters can be stored in a database and modified without direct manipulation of the GP model. Real, general integer and binary variables are allowed and nonlinear programming is supported. In a very small system, the goal programming could possibly be carried out using one of the spreadsheet programmes, such as Microsoft Excel, thus eliminating the need for additional software. The model has the form:

$$
\operatorname{Min} \sum_{i=1}^{k}\left(\left(\alpha_{i} n_{i}+\beta_{i} p_{i}\right) / T_{i}\right)
$$

Subject to:

$$
\begin{aligned}
& f_{i}(x)+n_{i}- p_{i}=T_{i} \\
& x \in F \\
& x \geq 0 \quad n \geq 0 \quad p \geq 0
\end{aligned}
$$

Where: $\alpha_{\mathrm{i}}$ and $\beta_{\mathrm{i}}$ are the weighting factors of the negative and positive deviations, $\mathrm{n}_{\mathrm{i}}$ and $\mathrm{p}_{\mathrm{i}}$, from the target value, $T_{i}$, of the attribute $i$ being considered. $F$ is the feasible set for the variable $x$. By setting $\alpha$ and $\beta$ to zero, a particular objective can be eliminated from the process without editing the template model. Dividing the sum of the weighted deviations by the target value results in a perunit comparison of the deviations rather than an absolute value comparison, which would be distorted by differences in magnitude and units.

Typically, $\mathrm{x}$ will represent; the demand on each route, the total number of each type of vehicle available, the policy frequency on various routes, the target fare on various routes, target vehicle load factors and the total available subsidy budget. Pollution, fuel, employment and other issues can be added to the model. The evaluation will seek the best compromise of vehicle assignment to policy objectives and technical constraints in an environment where it is not possible to satisfy all the demands. For example, one large and cheaper vehicle may be assigned to a route rather than the four smaller vehicles needed to meet the desired minimum frequency because of a funding problem. Further research will be conducted into including demand as a function of vehicle type and calibre in the GP model. Ultimately, the GP model is a "what if" tool for the planner, who can evaluate different policy proposals simply by changing one or two values in a database file. For example, what if a new vehicle type was to become available - how would system performance be affected?

The output of the model includes the number of vehicles of each type required on each route and the frequency of these vehicles. The shortfall and excess provision of any attribute is reported and the process results include cost information for each route, along with estimates of the economic fare required to operate the route. 


\section{TESTING THE RESULTS}

The output of the goal-programming model is added to the potential route database, which also has all the data necessary to create the batch input file for entering the transit lines into the Emme/2 model. The resulting route definitions are then converted to a suitable format for input to the Emme/2 model. This process has been coded as an option in the route extraction program.

A standard transit assignment is now performed. From this assignment, a reassessment of the transit network performance with boarding and alighting information as well as a graphic representation of the transit network is obtained. All the other Emme/2 transit assignment features can be used for more detailed analysis of the proposed system. Further refinements can be initiated based on the results of the transit assignment.

\section{CONCLUSIONS}

A brief outline of how Emme/2 or a similar network model can be used, in conjunction with a separate database and goal-programming model, to develop a set of fully described transit lines on a general transport network has been presented.

The modelling process seeks to satisfy the desires of customers and potential customers of the transit system whilst taking into account the practicalities of providing the service in a multioperator transport environment. The method also seeks to take into account the socio-economic, political and environmental objectives of the community being served by the transport service, seeking a compromise between various conflicting objectives.

The approach presented here does not claim to be all encompassing, but rather offers a concept, which can readily be adapted to the resources and goals of a region and which can be implemented without recourse to expensive specialist software other than the network model which is already well supported in South Africa.

\section{REFERENCES}

Elgar, A. \& Kfir, G. 1992. Computer aided bus route planning. 1st European EMME/2 Users' Conference, London, U.K.

Hasselström, D. 1981. Public Transportation Planning - a mathematical programming approach. Doctoral thesis, University of Gothenburg, Sweden.

INRO Consultants Inc. 1998. Emme/2 User's Manual: Release 9. Montreal, Canada.

LINDO Systems Inc. 1999. Demo Lingo/PC Release 6.0. Chicago, USA.

Romero, C. 1991. Critical issues in goal programming. Pergamon Press, UK. 


\title{
A PRACTICAL TRANSIT NETWORK DESIGN METHOD
}

\author{
W R Duff-Riddell \\ Institute for Transport Technology, Department of Civil Engineering, University of Stellenbosch, \\ Private Bag X1, Matieland, 7602, South Africa.
}

\section{Curriculum Vitae}

Wayne Duff-Riddell is a research assistant with the Institute for Transport Technology at the University of Stellenbosch. He has a master's degree in Civil Engineering and is now busy with research for his $\mathrm{Ph} . \mathrm{D}$., focusing on the design and management of public transport systems. He has presented papers at local and international conferences in this field.

$\mathrm{He}$ is a professional engineer and a member of the South African Road Federation where he is on the Traffic Engineering and Road Safety Committee. 